\title{
Caracterización fenotípica y genotípica de la deficiencia de glucosa-6-fosfato deshidrogenasa en Argentina. Estudio retrospectivo y descriptivo Phenotypic and genotypic characterization of glucose-6-phosphate dehydrogenase deficiency in Argentina. Retrospective and descriptive study
}

\author{
Bioq. Silvia Eandi Eberle", Bioq. Carolina Pepe ${ }^{a}$, Lic. Alejandro Chavesa, Bioq. Fernando Aguirrea, \\ Bioq. Berenice Milanesio ${ }^{a}$ Bioq. Diego Fernándeza , Dra. Vanesa Ávalos Gómeza , Dra. Gabriela Sciuccatia, \\ Dra. Lilian A. Díaz ${ }^{a}$ Dra. Andrea Candas ${ }^{a}$ Dra. Carolina Cervio ${ }^{a}$, Dra. Mariana Bonduel y Dra. Aurora Feliu Torres ${ }^{a}$
}

\section{RESUMEN}

La deficiencia de glucosa-6-fosfato deshidrogenasa es la enzimopatía eritrocitaria causada por mutaciones en el gen $G 6 P D$, cuya herencia está ligada al cromosoma X. Se analizan las características clínicas y de laboratorio de 24 individuos con deficiencia de G6PD durante 25 años. La edad mediana al momento del diagnóstico fue 10,2 años (rango: 0,6-56,4). El $54,2 \%$ de los pacientes fueron asintomáticos, mientras que el $25 \%$ presentó anemia hemolítica crónica no esferocítica; el $12,5 \%$, ictericia neonatal y anemia hemolítica posinfecciones, y el $8,3 \%$, anemia hemolítica aguda pos ingesta de habas. Los 24 pacientes estudiados presentaron variantes descritas previamente en la literatura. Las características clínicas observadas estuvieron acordes con las variantes encontradas. Veintiuna mujeres, pertenecientes a la rama materna de los individuos afectados, pudieron ser identificadas por biología molecular como portadoras de la deficiencia, por lo que recibieron el consejo genético correspondiente.

Palabras clave: errores innatos del metabolismo, glucosa-fosfato deshidrogenasa, técnicas de diagnóstico molecular.

http:/ / dx.doi.org/10.5546/ aap.2019.267

Texto completo en inglés:

http: / / dx.doi.org/10.5546/ aap.2019.eng.267

Cómo citar: Eandi Eberle S, Pepe C, Chaves A, Aguirre F, et al. Caracterización fenotípica y genotípica de la deficiencia de glucosa6-fosfato deshidrogenasa en argentina. Estudio retrospectivo y descriptivo. Arch Argent Pediatr 2019;117(4):267-270. a. Hospital de Pediatría "Prof. Dr. Juan P. Garrahan.

\section{Correspondencia:}

Bioq. Silvia Eandi Eberle: seandi@garrahan.gov.ar

Financiamiento: Ninguno.

Conflicto de intereses: Ninguno que declarar.

Recibido: 5-9-2018

Aceptado: 18-2-2019

\section{INTRODUCCIÓN}

La glucosa-6-fosfato deshidrogenasa (G6PD) cataliza la primera reacción en la vía de las pentosas fosfato, que aporta nicotinamida adenina dinucleótido fosfato reducido (NADPH), principal poder reductor de todas las células. El NADPH generado es crucial para prevenir el daño oxidativo a través del mantenimiento de los niveles intraeritrocitarios de glutatión. ${ }^{1} \mathrm{La}$ deficiencia de G6PD afecta a, aproximadamente, 500 millones de personas en el mundo y es la enzimopatía eritrocitaria más frecuente. Su área de distribución coincide con la del paludismo., ${ }^{2,3}$

Las mutaciones en el gen G6PD (Xq28) determinan variantes proteicas con diferentes niveles de actividad enzimática, asociadas a una amplia gama de fenotipos clínicos y bioquímicos.

Las manifestaciones clínicas en los individuos deficientes son muy variables: pueden ser asintomáticos o presentar ictericia neonatal, anemia hemolítica aguda (AHA) desencadenada por agentes infecciosos, drogas y/o ingesta de habas, o anemia hemolítica crónica no esferocítica (AHCNE). Según la clasificación de la Organización Mundial de la Salud (OMS), las variantes que ocasionan la AHCNE son designadas como clase I, mientras que aquellas que determinan episodios hemolíticos en relación con estrés oxidativo (infecciones, drogas o ingesta de habas) se designan como clase II o III. Las variantes tipo IV presentan actividad enzimática normal. Luzzato y Poggi proponen unificar las clases II y III, dado que todas estas variantes tienen una actividad enzimática menor del $30 \% .^{2-4}$

El objetivo de este trabajo es presentar las características clínicas y de laboratorio convencional y molecular de individuos con deficiencia de G6PD diagnosticados en el Servicio de Hematología-Oncología del Hospital de Pediatría "Prof. Dr. Juan P. Garrahan". 


\section{PACIENTES Y MÉTODOS}

Se realizó un estudio retrospectivo, descriptivo, longitudinal de 24 individuos (23 varones y 1 mujer) pertenecientes a 14 familias no relacionadas, con diagnóstico de deficiencia de G6PD, que consultaron entre marzo de 1991 y octubre de 2016. Seis individuos, de sexo masculino, fueron previamente reportados. ${ }^{5}$

El diagnóstico se basó en el estudio de patología eritrocitaria realizado al propósito y a su grupo familiar. El estudio incluyó la evaluación clínica, hemograma completo (Sysmex XS 800i, Sysmex Corporation, Kobe, Japan), examen del frotis de sangre periférica, recuento reticulocitario, electroforesis de hemoglobina semiautomatizada en gel de agarosa a $\mathrm{pH}$ alcalino, electroforesis capilar (Sebia, Lisses, Évry, France), cuantificación de hemoglobina $\mathrm{A}_{2}$ por cromatografía de intercambio aniónico (Helena), cuantificación de hemoglobina F por desnaturalización alcalina, prueba de resistencia osmótica eritrocitaria, metabolismo del hierro. El diagnóstico de la deficiencia de G6PD se realizó mediante la prueba de Brewer y la cuantificación de la actividad enzimática (Trinity Biotech). ${ }^{4}$

Para la realización del estudio molecular, se aisló ácido desoxirribonucleico (ADN) de leucocitos totales de sangre periférica por el método de precipitación salina. La presencia de mutaciones puntuales y/o pequeñas inserciones- deleciones en el gen G6PD fue evaluada por reacción en cadena de la polimerasa (polymerase chain reaction; PCR, por sus siglas en inglés), secuenciación en la que se utilizó un secuenciador automático ABI PRISM ${ }^{\circledR} 3130$ y los primers y estrategia descritos previamente. ${ }^{6}$

El estudio fue aprobado por el Comité Revisor de Investigación del Hospital de Pediatría "Prof. Dr. Juan P. Garrahan". Los pacientes y/o sus progenitores firmaron el consentimiento correspondiente en el momento de realizar el primer estudio de eritropatía.

\section{RESULTADOS}

En el período de estudio, se diagnosticaron 46 individuos deficientes (45 varones y 1 mujer). Solo en 24 individuos, con mediana de edad de 10,2 años y rango de 0,6-56,4, fue posible la caracterización genotípica. La Tabla 1 muestra las características de la población incluida en el estudio.

Seis pacientes (de \#1 a \#6), pertenecientes a 4 familias no relacionadas, presentaron AHCNE con una actividad enzimática menor del $10 \%$ y variantes moleculares descritas en la literatura como clase I. Los pacientes \#7, \#8 y \#9 presentaron ictericia neonatal, requerimiento transfusional ante episodios infecciosos y una actividad enzimática menor del $10 \%$ asociada a la presencia de la variante Mediterránea

TABLA 1. Características clínicas y de laboratorio

\begin{tabular}{|c|c|c|c|c|c|c|}
\hline \#Pt (Flia.) & Sexo & Clase & $\mathrm{AE} \%$ & Clínica & Mutación NP_001035810.1 & Variante \\
\hline \#1 (1) & M & I & $<10$ & AHCNE & p.(Ser332Phe) & Quilmes \\
\hline$\# 2(2)$ & M & I & $<10$ & AHCNE & p.(Pro409Gln) & Merlo \\
\hline$\# 3, \# 4, \# 5$ (3) & M & I & $<10$ & AHCNE & p.(Phe381Ile) & New York \\
\hline \#6 (4) & M & I & $<10$ & AHCNE & p.(Arg387His) & Beverly Hills \\
\hline \#7 (5) & M & II & $<10$ & ICN AHA & & \\
\hline \#8 (6) & M & II & $<10$ & ICN AHA & p.(Ser188Phe) & Mediterránea \\
\hline \#9 (7) & M & II & $<10$ & ICN AHA & & \\
\hline$\# 10(8)$ & M & III & $10-30$ & ASINT. & & \\
\hline$\# 11, \# 12, \# 13$ (9) & M & III & $10-30$ & ASINT. & & \\
\hline \#14 (10) & M & III & $10-30$ & ASINT. & & \\
\hline \#15 (11) & M & III & $10-30$ & ASINT. & p.(Val68Met, Asn126Asp) & $\mathrm{A}^{-202}$ \\
\hline \#16 (12) & M & III & $10-30$ & ASINT. & & \\
\hline \#17 (13) & M & III & $10-30$ & ASINT. & & \\
\hline$\# 18$ (13) & M & III & $10-30$ & AHA & & \\
\hline \#19, \#20 (14) & M & III & $10-30$ & ASINT. & & \\
\hline \#21 (12) & M & III & $10-30$ & AHA & p.(Asn126Asp, Leu323Pro) & $\mathrm{A}^{-968}$ \\
\hline$\# 22, \# 23(12)$ & M & III & $10-30$ & ASINT. & & \\
\hline \#24 (12) & $\mathrm{F}$ & III & $10-30$ & ASINT. & p.(Val68Met, Leu323Pro) & $\mathrm{A}^{-202} / \mathrm{A}^{-968}$ \\
\hline
\end{tabular}

Flia.: familia; M: masculino; F: femenino; AE: actividad enzimática; ASINT.: asintomático; AHCNE: anemia hemolítica crónica no esferocítica; ICN: ictericia neonatal; AHA: anemia hemolítica aguda; NP: número de referencia de la secuencia proteica. 
descrita en la literatura como variante clase II. Nueve pacientes pertenecientes a 6 familias presentaron la variante molecular $\mathrm{A}^{-202}$ (clase III) y una actividad enzimática de entre el $10 \%$ y el $30 \%$. Siete pacientes de este grupo (de \#10 a \#16) fueron asintomáticos y la deficiencia de G6PD fue detectada en el contexto del estudio familiar de patología eritrocitaria. Los dos pacientes restantes (\#17 y \#18) eran hermanos y presentaron manifestaciones clínicas diferentes: uno asintomático y el otro con crisis hemolítica pos ingesta de habas. En el grupo de los 5 pacientes con la variante $\mathrm{A}^{-968}$, dos hermanos asintomáticos fueron detectados por una prueba de Brewer positiva en el marco de un estudio familiar de hemoglobinopatías. Los otros 3 pacientes (\#21, \#22, \#23) con la variante $A^{-968}$ eran hermanos. El caso índice (\#21) presentó una crisis hemolítica pos ingesta de habas y sus hermanos eran asintomáticos (\#22, \#23). La última paciente (\#24), mujer asintomática, con prueba de Brewer positiva y doble heterocigota para las variantes $A^{-968} / A^{-202}$, era la madre de 4 pacientes, 3 genotipificados con la variante $A^{-968 \mathrm{w}}$ (\#21, \#22 y \#23) y uno con la variante $A^{-202}$ (\#16).

A partir de la secuenciación e identificación de la variante molecular involucrada, fue investigada en todas las mujeres de la rama materna disponibles, madres, hermanas, tías y primas. Así, sobre un total de 29 mujeres estudiadas pertenecientes a 9 familias, 21 pudieron identificarse como portadoras heterocigotas.

\section{DISCUSIÓN}

La G6PD es una enzima constitutiva (housekeeping) vital para las células y la ausencia completa de actividad enzimática sería incompatible con la vida.

La G6PD cataliza el primer paso de la vía de las pentosas. Esta vía metabólica produce $\mathrm{NADPH}$, que permite mantener, en las células, un medioambiente adecuado reducido, especialmente en los eritrocitos.

La deficiencia de G6PD presenta una gran heterogeneidad clínica y molecular. Se han reportado, a nivel mundial, más de 200 variantes del gen G6PD asociadas a sintomatología muy leve, como ocurre con la mayoría de los pacientes con la variante $\mathrm{A}^{-}$, hasta grave, como se observa en la anemia hemolítica grave presente en los pacientes con la variante Mediterránea o AHCNE en individuos con la variante Guadalajara. ${ }^{2}$

En nuestra serie de 24 pacientes estudiados con pruebas convencionales y moleculares, se pudo correlacionar la clínica, la actividad enzimática y el defecto molecular. En los pacientes con AHCNE y una actividad enzimática $<10 \%$, los estudios moleculares identificaron 4 variantes pertenecientes a la clase I de la OMS, 2 de estas (variantes Merlo y Quilmes) fueron descritas recientemente por nuestro laboratorio. ${ }^{7}$

Tres de las variantes encontradas (Merlo, New York y Beverly Hills) se encuentran en el exón 10 y afectan aminoácidos que participan en la dimerización; de esta manera, alteran un paso esencial para la existencia de actividad catalítica. Estas mutaciones rompen los contactos entre las dos subunidades o desorganizan la estructura en la interfase por introducción de aminoácidos con carga eléctrica diferente o de residuos con tamaños distintos. ${ }^{7,8}$

En la variante Quilmes, la sustitución de Ser 332Phe podría generar un cambio conformacional en el loop de acceso al sitio activo, lo que afectaría la actividad de la enzima al modificar la unión al sustrato glucosa-6-fosfato (G6P). ${ }^{7}$

La variante Mediterránea, presente en 3 pacientes, se localiza en el exón 6 . El nuevo aminoácido, que se codifica, aumenta la afinidad de la enzima por la -G6P. Esta característica podría explicar por qué, a pesar de ser pacientes gravemente deficientes, no presentan AHCNE y son considerados clase II. ${ }^{2}$

La variante $\mathrm{A}^{-202}$ es consecuencia de dos cambios de tipo missense que afectan los exones 4 y 5 . A partir del estudio tridimensional de la molécula, se observa que las posiciones de los aminoácidos afectados se aproximan de tal manera que, espacialmente, ambos residuos quedan muy cercanos entre sí. En función de esto, dichos cambios actuarían sinérgicamente y causarían la deficiencia, por disrupción de la estructura proteica, y afectarían el sitio de dominio de la coenzima. ${ }^{9}$

Por último, en la variante $\mathrm{A}^{-968}$, se observa un cambio múltiple tipo missense, que afecta los exones 5 y 9. Esta variante determina las sustituciones de aminoácidos Asn126Asp. y Leu323Pro. de la molécula. Se postula que, como consecuencia de estos cambios moleculares, tanto la actividad como la estabilidad enzimática se encuentran comprometidas. Probablemente, el efecto más preponderante se debería a la sustitución Leu323Pro en la caracterización de la variante $\mathrm{A}^{-968 .}{ }^{-10}$

En conclusión, la confirmación de la existencia de una correlación fenotipo-genotipo permitió diseñar un algoritmo de estudio molecular del gen 
G6PD en función de las características clínicas de los pacientes estudiados. Además, la identificación de la alteración molecular presente en los varones deficientes posibilitó el consejo genético en las mujeres de las diferentes familias.

\section{REFERENCIAS}

1. Cappellini MD, Fiorelli G. Glucose-6-phosphate dehydrogenase deficiency. Lancet. 2008; 371(9606):64-74.

2. Luzzatto L, Poggi V. Glucose-6-phosphate dehydrogenase deficiency. In: Orkin SH, Nathan D, Fisher DE, Ginsburg $\mathrm{D}$, et al (eds.). Nathan and Oski's Hematology of Infancy and Childhood. $8^{\text {th }}$ ed. Philadelphia: Saunders Elsevier; 2015. Págs.609-29.

3. Beutler E. G6PD: population genetics and clinical manifestations. Blood Rev. 1996; 10(1):45-52.

4. Roper D, Layton M. Investigation of the hereditary haemolyticanaemias:membraneand enzymeabnormalities. In:Lewis SM, Bain BJ, Bates I(eds.). Dacie and Lewis' Practical Haematology. $10^{\text {th }}$ ed. Philadelphia: Churchill Livingston; 2006.Págs.205-37.
5. Eandi Eberle S, García Rosolen N, Urtasum C, Sciucati G, et al.Deficiencia de glucosa-6-fosfato deshidrogenasa.Serie de casos clínicos. Arch Argent Pediatr. 2011; 109(4):354-7.

6. Poggi V, Town M, Foulkes NS, Luzzatto L. Identification of a single base change in a new human mutant glucose6-phosphate dehydrogenase gene by polymerase-chain reaction amplification of the entire coding region from genomic DNA. Biochem J. 1990; 271(1):157-60.

7. Chaves A, Eberle SE, Defelipe L, Pepe C, et al. Two novel DNA variants associated with glucose-6-phosphate dehydrogenase deficiency found in Argentine pediatric patients. Clin Biochem. 2016; 49(10-11): 808-10.

8. Lee J, Park J, Choi H, Kim J, et al. Genetic Profiles of Korean Patients With Glucose-6-Phosphate Dehydrogenase Deficiency. Ann Lab Med. 2017; 37(2):108-16.

9. Naylo CE, Rowland P, Basak AK, Gover S, et al. Glucose 6-Phosphate Dehydrogenase Mutations Causing Enzyme Deficiency in a Model of the Tertiary Structure of the Human Enzyme. Blood. 1996; 87(7):2974-82.

10. Ramírez-Nava EJ, Ortega-Cuellar D, Serrano-Posada H, González-Valdez A, et al. Biochemical Analysis of Two Single Mutants that Give Rise to a Polymorphic G6PD A-Double Mutant. Int J Mol Sci. 2017; 18(11):E2244.

\section{Archivos hace 75 años}

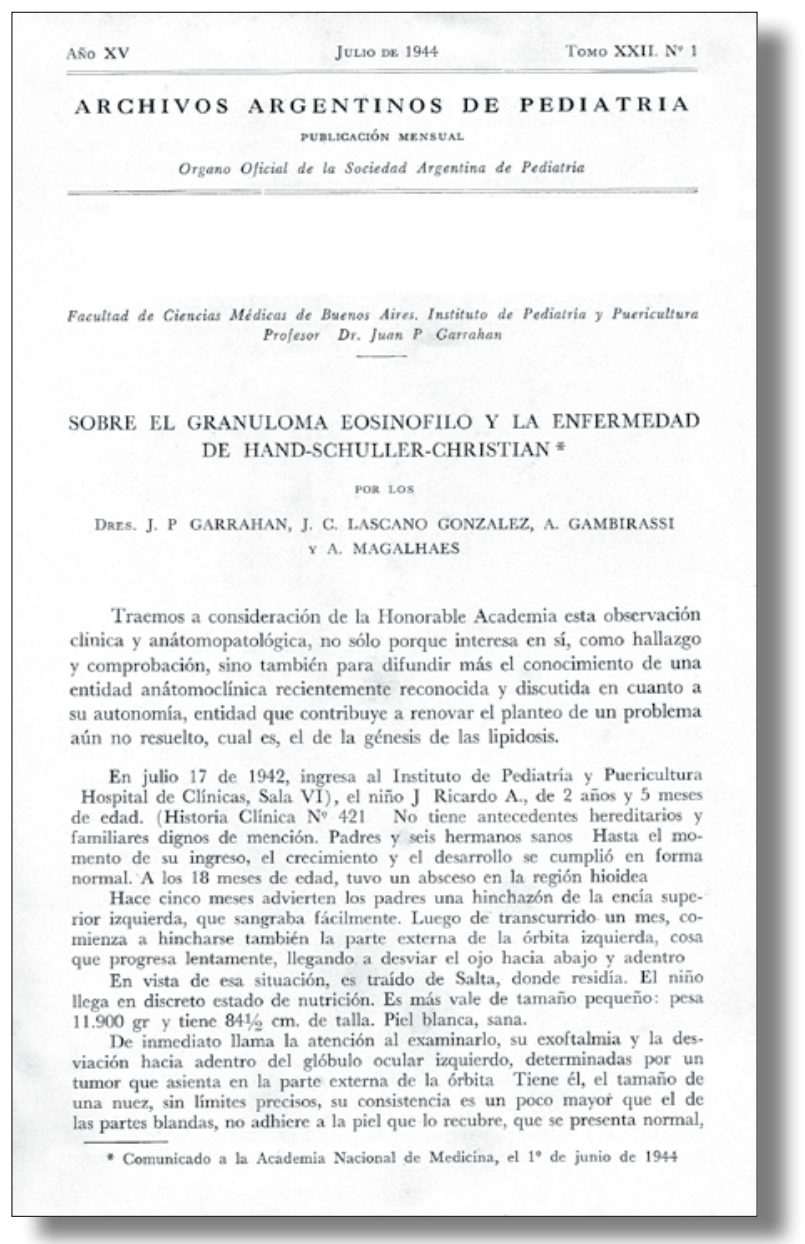

El texto completo se encuentra disponible en la versión electrónica de este número. 